\title{
EDITOR'S
}

\section{Models for investigation in Urology: Rats, Pigs and Dogs. Importance of basic research in penile reconstruction and bladder overactivity.}

The May-June 2014 issue of the International Braz J Urol presents original contributions with a lot papers in different fields. The papers come from many different countries such as Brazil, USA, China, Italy, Korea, Germany, Greece, Iran and Turkey, and as usual the editor's comment highlights some papers. In this number we can observe some papers about basic research in Peyronie's disease and bladder overactivity. Several medical treatments are suggested to Peyronie's disease and overactive bladder. A lot of surgical techniques exist for the treatment of Peyronie's disease and penile reconstruction but the best surgical treatment still is under discussion. These two diseases still represent a clinical challenge for the urologist. We summarize three interesting papers about basic research in these topics below.

Doctor Salehipour and collegues, from the Departments of Urology, from the Shiraz university from Shiraz, Iran performed on page 105 an interesting study to evaluate the efficacy of human amniotic membrane (AM) grafting in the canine penile tunica albuginea defect; the authors developed an animal model as the first step toward an innovating new method for the treatment of Peyronie's disease. The authors concluded that the amniotic membrane can be used as a suitable substitution for tunica albuginea. It is safe, inexpensive, biodegradable, and available and may be used for the treatment of Peyronie's disease, penile cancers, congenital penile deformities, and penile reconstructive surgery.

Doctor Dambros and collegues from the department of urology of the University Hospital Maastricht and the School of medicine Sao Leopoldo, Sao Paulo, Brazil performed on page 113 a elegant study about the involuntary detrusor contractions. The authors studied the possibility to induce the muscle overactivity with oxidative stress using hypochlorous acid ( $\mathrm{HOCl})$. The authors concluded that an oxidative stressor, like $\mathrm{HOCl}$, is capable of inducing smooth muscle overactivity. This model can be used for the development and testing of new treatment modalities for the overactive detrusor. Furthermore, this study provides evidence for a causal relationship between oxidative stress and detrusor overactivity.

Doctor Zhang and collegues from the department of urology and Pathology from Shandong Tumor Hospital, Jinan, performed on page 119 a study about a model for establishment and evaluation of detrusor overac- tivity in female Wistar rats. The authors ligated the perineal urethra of female Wistar rats and then performed filling cystometry. They observed that bladder weight increased significantly in detrusor overactivity rats and concluded that ligating perineal urethra and filling cystometry with intra-urethral cannula approach is a simple and easily reproducible method to establish and evaluate the model of detrusor overactivity in rats.

Luciano A. Favorito, MD, PhD

Associated Professor of Urogenital Research Unit State University from Rio de Janeiro - UERJ 